\title{
Efficient Infection of Nicotiana benthamiana by Tomato bushy stunt virus Is Facilitated by the Coat Protein and Maintained by p19 Through Suppression of Gene Silencing
}

\author{
Feng Qu and T. Jack Morris \\ School of Biological Sciences, University of Nebraska-Lincoln, 348 Manter Hall, Lincoln, Nebraska 68588-0118, U.S.A. \\ Submitted 30 August 2001. Accepted 9 November 2001.
}

\begin{abstract}
Tomato bushy stunt virus (TBSV) is one of few RNA plant viruses capable of moving systemically in some hosts in the absence of coat protein (CP). TBSV also encodes another protein (p19) that is not required for systemic movement but functions as a symptom determinant in Nicotiana benthamiana. Here, the role of both CP and p19 in the systemic spread has been reevaluated by utilizing transgenic $N$. benthamiana plants expressing the movement protein (MP) of Red clover necrotic mosaic virus and chimeric TBSV mutants that express CP of Turnip crinkle virus. Through careful examination of the infection phenotype of a series of mutants with changes in the $C P$ and p19 genes, we demonstrate that both of these genes are required for efficient systemic invasion of TBSV in $N$. benthamiana. The CP likely enables efficient viral unloading from the vascular system in the form of assembled virions, whereas p19 enhances systemic infection by suppressing the virus-induced gene silencing.
\end{abstract}

A critical step in viral pathogenicity is systemic plant infection, when a virus spreads from the initial infection site to distal parts of the plant through the vascular system (Carrington et al. 1996; Seron and Haenni 1996). This movement process is believed to involve both viral-specific and host-specific functions that are distinct from those involved in cell-to-cell movement (Schaad and Carrington 1996). Most RNA plant viruses require expression of the viral coat protein $(\mathrm{CP})$ for efficient systemic movement. In some cases the assembled virus particles are also necessary (Dolja et al. 1995; Taliansky and Garcia-Arenal 1995; Vaewhongs and Lommel 1995). Tomato bushy stunt virus (TBSV) belongs to a unique class of RNA viruses that do not require $\mathrm{CP}$ for systemic infection in certain hosts. Scholthof and associates (1993) first reported that TBSV was able to spread systemically in Nicotiana clevelandii and $N$. benthamiana plants in the absence of $\mathrm{CP}$ expression, albeit with some delay, and cause significant systemic disease symptoms. TBSV is the type member of the Tombusviridae family consisting of several distinct but closely related genera of spherical RNA plant viruses. CP, most likely in the form of assembled viruses, is known to be required for the systemic movement of two other members of the same family, Red clover necrotic mosaic virus (RCNMV) and Turnip crinkle virus

Corresponding author: Jack Morris; Telephone 402-472-6676; FAX: 402-472-2083; Email: jmorris@unlnotes.unl.edu
(TCV) (Heaton et al. 1991; Li et al. 1998; Vaewhongs and Lommel 1995). Hence, the movement behavior reported for TBSV was somewhat unusual.

TBSV is a 30-nm icosahedral virus with a single-stranded, positive sense RNA genome of about $4.8 \mathrm{~kb}$ that encodes five open reading frames (ORFs) (Hearne et al. 1990). The $5^{\prime}$ proximal ORF (p33) and its readthrough product (p92) encode the viral RNA-dependent RNA polymerase (Oster et al. 1998). The $\mathrm{CP}$ ORF lies in the middle of the genome and is translated from a subgenomic RNA (sgRNA 1). A smaller sgRNA (sgRNA 2), derived from $3^{\prime}$ fourth of the genome, is responsible for the expression of two nested genes, p19 and p22. P22 is the viral movement protein (MP), as it has been shown to be necessary for cell-to-cell movement and, hence, systemic movement in all hosts tested (Scholthof et al. 1993, 1995a). Interestingly, p19, although not required for cell-to-cell movement, was shown to be necessary for systemic movement in some hosts (e.g., spinach) but not in others, including $N$. benthamiana (Chu et al. 2000; Scholthof et al. 1995a).

While these studies demonstrated that neither CP nor p19 were necessary for systemic spread of TBSV viral RNA in $N$. benthamiana, systemic invasion was delayed in plants inoculated with the CP-defective mutants, and the symptoms were generally milder than in wild-type infections, implying that absence of CP impaired systemic movement. On the other hand, P19 was found to be primarily a symptom determinant responsible for causing the characteristic necrotic symptoms associated with TBSV infections (Scholthof et al. 1995b). More recently, it has been shown to be involved in suppression of posttranscriptional gene silencing (PTGS) of transgenes in $N$. benthamiana (Carrington et al. 2001; Voinnet et al. 1999). Therefore, the necrosis associated with expression of p19 might be the indirect result of the suppression of the host defense process associated with PTGS (Chu et al. 2000). However, direct evidence that p19 functions as a suppressor of gene silencing in the process of TBSV infection is lacking.

In this study, we have reevaluated the role of both $\mathrm{CP}$ and p19 in the systemic invasion of $N$. benthamiana by TBSV. First, we were able to eliminate complications associated with mutagenesis of the nested p19 and p22 genes by utilizing transgenic $N$. benthamiana plants expressing the RCNMV MP to complement the cell-to-cell movement function. Secondly, we were able to replace the TBSV CP gene with a functionally equivalent $\mathrm{CP}$ gene from the structurally related TCV. Together, these strategies permitted a more thorough assessment of the systemic infection process using mutants defective in the TBSV CP and p19 functions. We conclude that both of these 
genes participate in efficient systemic TBSV infection of $N$. benthamiana. In addition, our results suggest that assembled virions are likely important for the effective unloading of viruses from the vascular system into the leaf mesophyll. Moreover, we provide direct evidence that p19 primarily enhances systemic invasion by suppressing the host PTGS responsible for eliminating viral RNAs in the infected plants.
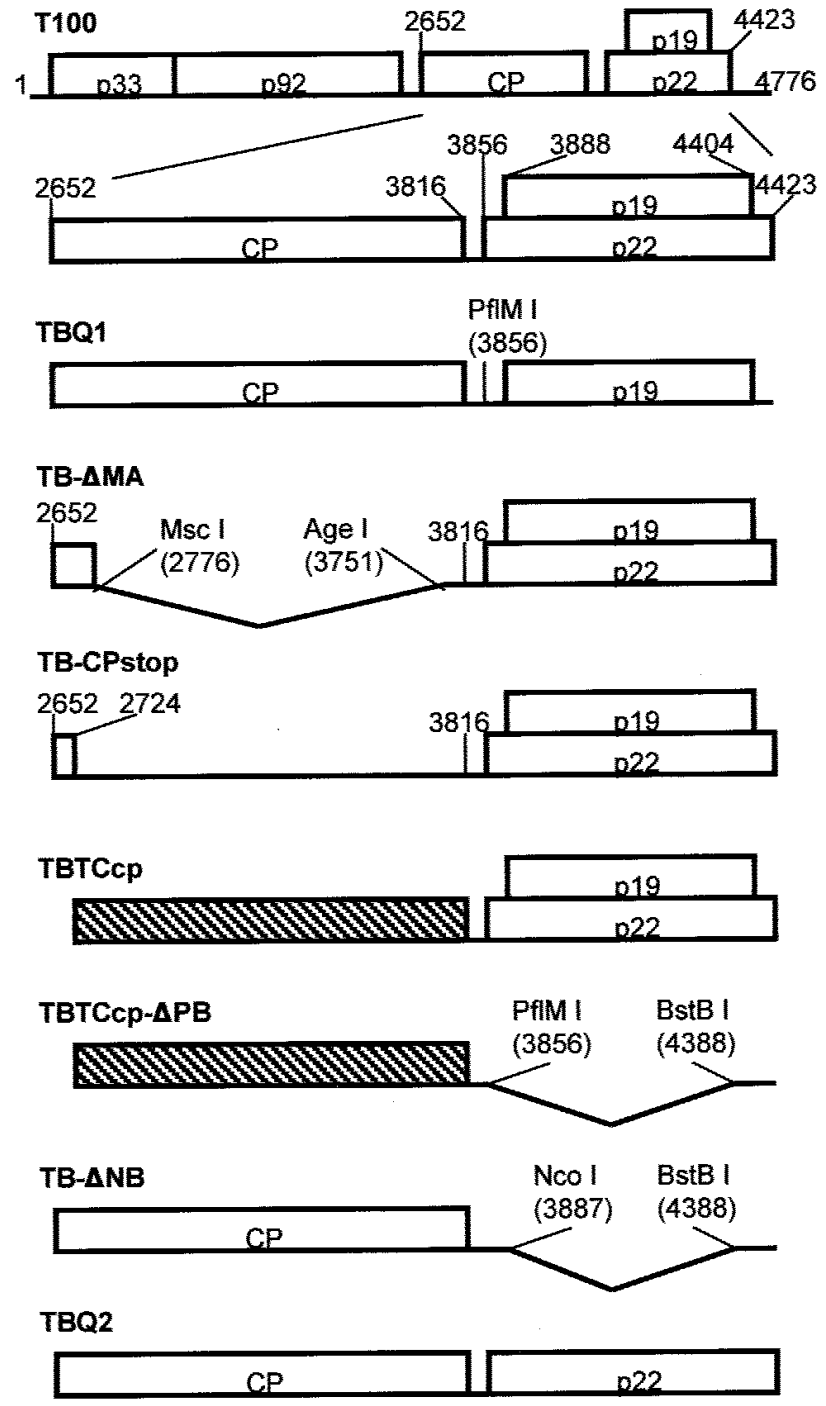

TB- $\triangle M A+T B-\Delta N B$

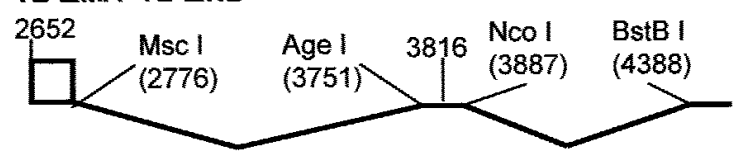

TBQ2-CPstop

$2652 \quad 2724$

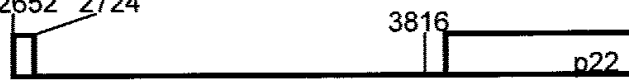

Fig. 1. Schematic representation of the Tomato bushy stunt virus (TBSV) genome and of the mutant genomes used in this study. The top drawing shows the TBSV genome (T100) with the five open reading frames (ORFs) represented as boxes and the numbers representing nucleotide locations. The portion of the genome encoding the coat protein (CP) and the $\mathrm{p} 22 / \mathrm{p} 19$ ORF is expanded directly underneath. Diagrams of the alterations made in the various mutants designated on the left above each diagram are shown. A detailed description of each mutant is given in the text.

\section{RESULTS}

Cell-to-cell movement of TBSV with a defective p22 gene is complemented by transgenic $N$. benthamiana plants expressing RCNMV MP.

The TBSV $\mathrm{p} 22$ has been demonstrated to be responsible for cell-to-cell movement of viral RNA (Scholthof et al. 1995a). Several studies have shown that MPs of unrelated plant viruses are interchangeable (Cooper et al. 1996; De Jong and Ahlquist 1992). In particular, RCNMV MP has been shown to complement the MP functions of several different RNA viruses, including an insect virus (Dasgupta et al. 2001; GiesmanCookmeyer et al. 1995; Rao et al. 1998; Reade et al. 2001). The complete overlap of the p19 gene within the p22 gene (Fig. 1) constrains mutational analysis of p19 function. In order to uncouple the function of the two nested genes, we acquired transgenic $N$. benthamiana plants that expressed the RCNMV MP (MP+ plants) from S. A. Lommel and found that RCNMV MP complemented the cell-to-cell movement function of p22. In this experiment, mutant TBQ1, which has no p22 start codon (Fig. 1), was found to be unable to infect nontransgenic $N$. benthamiana (NT) plants, as expected. However, in MP+ plants inoculated with TBQ1, systemic symptoms typical of TBSV infection started to appear at 6 days postinoculation (dpi), which is a delay of only one day relative to plants inoculated with the wild-type TBSV transcript (T100). The initial symptoms for both infections included crinkling and vein clearing on the young apical leaves, quickly developing into veinal necrosis and leaf collapse (Fig. 2B, C, and D), followed by death of most of the inoculated plants within 14 dpi. RNA blot analysis revealed that accumulation of TBSV-specific RNAs in systemic leaves of wild-type-infected and TBQ1-infected MP+ plants were comparable (Fig. 3, lanes 2 and 3). Sequence analysis showed that the original mutation in TBQ1 was still present in the progeny RNAs (data not shown). These results demonstrate that the RCNMV MP complemented the cell-to-cell movement function of TBSV to near wild-type levels.

\section{Inefficient systemic movement of CP-defective mutants.}

Scholthof and associates (1993) showed that the absence of functional CP significantly delayed (by 3 to 5 days) but did not eliminate systemic movement of TBSV RNA in $N$. benthamiana. To determine the cause of the delay, we examined the infection phenotype of two mutants altered in the TBSV CP gene. TB- $\triangle \mathrm{MA}$ was created by deleting about $1 \mathrm{~kb}$ of the CP gene. TB-CPstop was created by introducing two consecutive stop codons into the CP ORF after the first 24 amino acids (Fig. 1). Each mutant was inoculated onto three NT and three $\mathrm{MP}+$ plants, and the symptoms were carefully compared to a similar set of plants inoculated with T100. Both mutants were found to cause a similar pattern of overall symptom development in both NT and MP+ plants that was distinctively different from normal systemic invasion by wild-type TBSV. The observations permitted two general conclusions. First, the CPcoding RNA itself did not contribute to TBSV pathogenesis in $N$. benthamiana (Szittya and Burgyan 2001). Second, the unusual pattern of spread of the CP mutants did not seem to be affected by the mode of expression of the MP.

The inoculated leaves of plants infected with the CP mutants showed signs of infection as early as 4 to $5 \mathrm{dpi}$, but the appearance of local symptoms was drastically different. While lesions on T100-inoculated leaves quickly expanded and turned necrotic, leading to leaf collapse at 9 to $10 \mathrm{dpi}$ (Fig. 2C), lesions caused by the CP mutants appeared smaller, expanded more slowly, and extended mostly along the primary veins, usually accompanied by necrosis of the affected primary veins (Fig. 2E). 


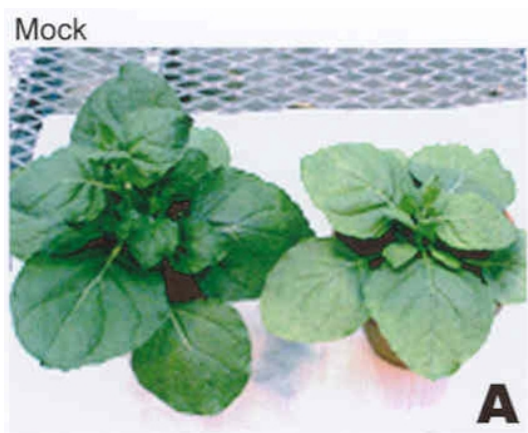

$\mathrm{MP}+/ \mathrm{T} 100,9 \mathrm{dpi}$

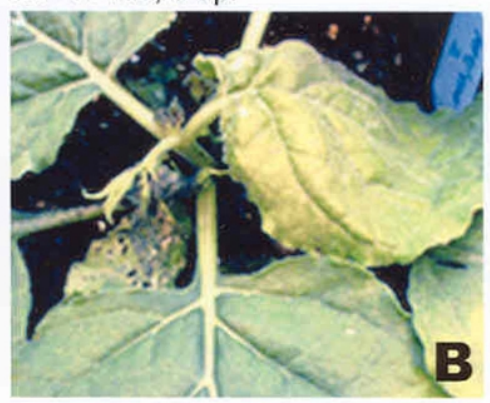

$\mathrm{MP}+/ \mathrm{TBQ} 1,9 \mathrm{dpi}$

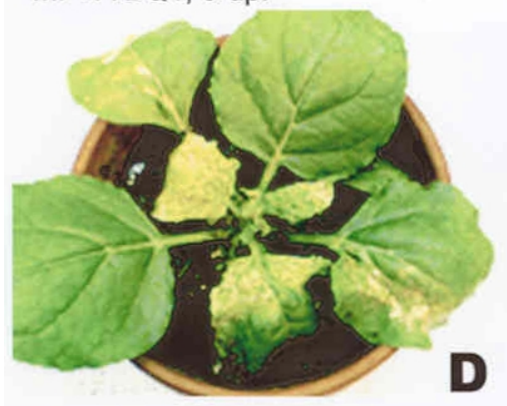

MP+/TBCPstop, IL, 9 dpi

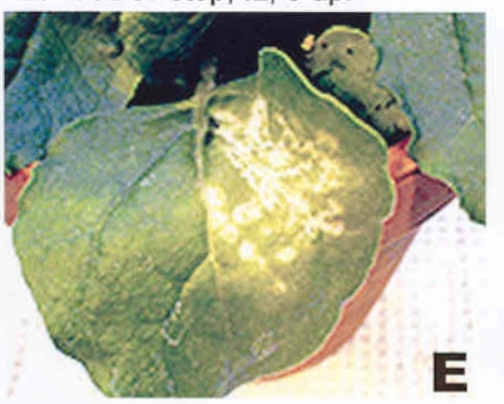

$\mathbf{E}$
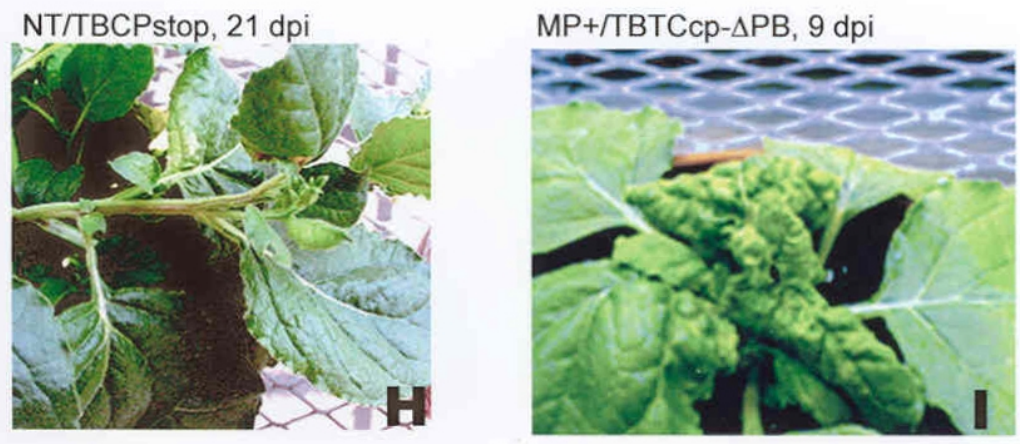

NT/TBCPstop, SL, 9dpi

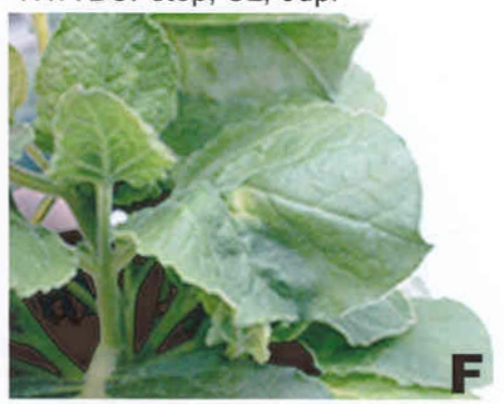

$\mathrm{MP}+/ \mathrm{T} 100, \mathrm{IL}, 9 \mathrm{dpi}$

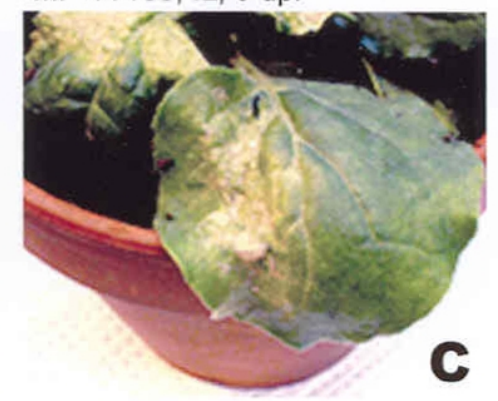

C

\section{=}

NT/TB- $\triangle M A, 21$ dpi
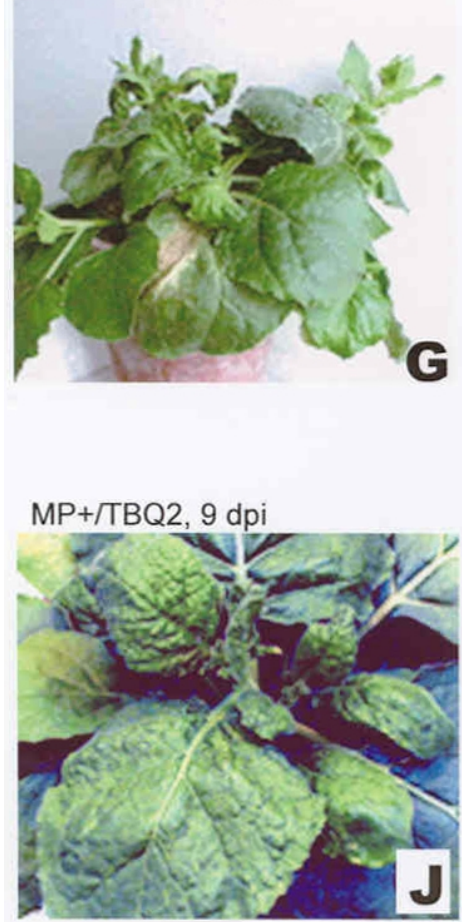
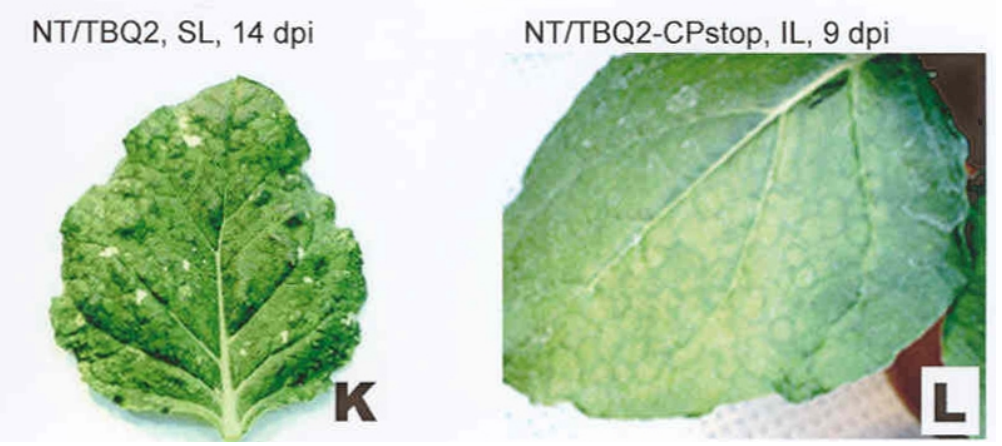

Fig. 2. Symptoms of Tomato bushy stunt virus (TBSV) T-100 and its mutants in Nicotiana benthamiana plants. The inoculated plants are either nontransgenic (NT) plants or transgenic plants expressing Red clover necrotic mosaic virus movement protein $\left(\mathrm{MP}^{+}\right)$. A, Mock-inoculated NT (left) and MP ${ }^{+}$ (right) plants. B, Wild-type TBSV infection (T-100) on an $\mathrm{MP}^{+}$plant at 9 days postinoculation (dpi), showing typical symptoms of systemic necrosis and apical collapse. C, Inoculated halfleaf of a T100-infected $\mathrm{MP}^{+}$plant at $9 \mathrm{dpi}$, showing typical necrosis and leaf collapse. D, Systemic symptoms of an $\mathrm{MP}^{+}$ plant at 9 dpi, after inoculation with the p22 defective mutant TBQ1. E, Inoculated leaf of an $\mathrm{MP}^{+}$plant inoculated with coat protein defective mutant TBCPstop at 9 dpi. Note the atypical development of necrosis along the leaf veins as compared with panel C. F, A systemic leaf of an NT plant inoculated with the TB-CPstop mutant at 9 dpi. Note the leaf curling and small region of necrosis near the vein. G, An NT plant showing a bushy appearance at 21 dpi, with the coat protein (CP) defective mutant TB- $\triangle$ MA. H, A closeup of a necrotic branch on an NT plant 21 dpi, with the CP defective mutant TBCPstop. I, Systemic symptoms of an $\mathrm{MP}^{+}$plant $9 \mathrm{dpi}$, with the truncated TBSV/TCV chimeric RNA, TBTCcp- $\Delta$ PB. J, Systemic symptoms of an MP ${ }^{+}$ plant 9 dpi, with p19 defective mutant, TBQ2. K, A systemic leaf of a TBQ2-inoculated NT plant at 14 dpi, showing signs of recovery. L, An inoculated leaf on an NT plant at $9 \mathrm{dpi}$, with the double mutant TBQ2-CPstop defective in expression of both the $\mathrm{p} 19$ and the $\mathrm{CP}$ genes. IL $=$ inoculated leaf and SL $=$ systemically infected leaf. 
The rate of systemic spread of the two mutants in both MP+ and NT plants was also quite variable and consistently delayed. The first signs of systemic infection by the TBCPstop mutant appeared at $6 \mathrm{dpi}$ on two plants, $8 \mathrm{dpi}$ on two other plants, and at $12 \mathrm{dpi}$ on the remaining two plants. The TB- $\Delta$ MA mutant behaved similarly, exhibiting signs of systemic infection on two plants at $7 \mathrm{dpi}$ and on the remaining four plants at $8 \mathrm{dpi}$. Most interestingly, the systemic symptoms on these plants were characteristically distinct from that of a typical TBSV infection. First, systemic symptoms rarely occurred on young apical leaves, with only 1 of the 12 plants showing apical symptoms as the first sign of systemic spread. The initial symptoms were most often observed on only one older leaf in each plant. Additional leaves of the plant were infected gradually and randomly, with infection often not becoming apparent until 3 or more days after the first symptomatic leaf appeared. Less than $40 \%$ of the leaves of the entire plant became symptomatic by 35 dpi. However, those infected leaves were always sink leaves, and the appearance of the systemically infected leaves was quite unique. These leaves were distorted or even twisted, with a curled, shortened, and chlorotic primary vein (Fig. 2F). A small chlorotic area would then develop in the vicinity of the vein and slowly expand into a 1- to 2-cm area of necrosis with the vein at its center. Mutant viral RNA could be readily detected in these symptomatic leaves (Fig. 3, lanes 4 and 5).

A third characteristic feature of the infections by CP-defective mutants was the necrosis of the vascular tissue. Necrotic streaks started to appear between 12 and 21 dpi on some stems and major branches of the plants, causing the entire plant to bend or twist. The deformed stems and branches, together with the mostly green and healthy-looking leaves on them, created a distinct bushy appearance (Fig. 2G and H). In a few cases, the leaf petioles went necrotic but still retained healthy-looking leaves. Individual necrotic branches were dissected and analyzed for the presence of viral RNA in various parts of the branch and leaves grown on the branch. The results of one such experiment, shown in Figure 4, demonstrated that viral RNA was confined to the stem and symptomatic leaves extended from the affected branch but was completely absent in asymptomatic leaves on the same branch.

The characteristic symptoms on the plants infected by both $\mathrm{CP}$ mutants suggested that the mutant viral RNAs were able to enter and spread efficiently in the vascular elements but apparently unable to exit the vascular tissue into leaf mesophyll cells in either the inoculated or systemically infected leaves. We further speculate that virion assembly might be necessary for efficient exit of viruses from vascular tissue.

\section{TCV CP can substitute for TBSV CP}

\section{in facilitating the systemic movement of TBSV RNA.}

We next wanted to directly address whether virion assembly, as opposed to some other effect of viral $\mathrm{CP}$, could be the primary factor contributing to the abnormal systemic movement phenotype of the TBSV CP mutants. In a previous study focused on viral RNA encapsidation, we produced a TBSV/TCV chimera (Fig. 1, TBTCcp) in which we replaced the CP gene of TBSV with that of the structurally related CP of TCV (Qu and Morris 1997). We showed that, in protoplast infections, TCV $\mathrm{CP}$ would package the subgenomic RNAs but not the genomic RNA of TBTCcp. We were also able to make the TBTCcp genome assembly competent by reducing the size of the genomic RNA to $4.2 \mathrm{~kb}$ with a deletion that eliminated most of the p19/p22 ORF (Fig. 1, mutant TBTCcp- $\triangle$ PB). These results indicated that the inability of TCV CP to package TBTCcp RNA ( $4.7 \mathrm{~kb}$ in size) was due to the RNA size limit imposed by TCV $\mathrm{CP}$ (TCV genomic RNA is $4.05 \mathrm{~kb}$ ). The demonstration that
$\mathrm{MP}+$ plants complemented the TBSV mutant defective in cellto-cell movement offered the opportunity to directly assess the role of assembly in the systemic spread using these chimeric viruses. Three NT and three MP+ plants were inoculated with each of the chimeric mutants. The TBTCcp mutant was unable to systemically infect NT plants, forming only small chlorotic spots on inoculated leaves at $6 \mathrm{dpi}$ that failed to enlarge for the duration of the experiment (35 dpi). This result was not unexpected because the replacement of the TBSV CP gene with the TCV CP gene would have deleted an important promoter element for the sgRNA 2 (Zhang et al. 1999), negatively affecting the expression of $\mathrm{p} 22$, and making TBTCcp inefficient at cellto-cell movement. Interestingly, the movement defect of TBTCcp was also not very efficiently complemented in MP+ plants as compared to the TBQ1 mutant. The initial signs of invasion appeared in each of the three inoculated plants as a single distorted leaf at 11, 14, and $21 \mathrm{dpi}$. All three plants remained relatively healthy, each with only a few distorted leaves by 35 dpi. Although much milder, the symptoms generally resembled those produced by the TBSV CP-defective mutants described previously.

The TBTCcp- $\triangle \mathrm{PB}$ mutant failed to infect NT plants. This was expected because of the absence of a functional p22 gene in this mutant. Most interesting, however, was the almost wildtype invasiveness of TBTCcp- $\triangle \mathrm{PB}$ in the MP+ plants. Systemic symptoms developed on $\mathrm{MP}+$ plants as early as $6 \mathrm{dpi}$ and were apparent on all three plants at 9 dpi. Furthermore, unlike the case of the CP mutants, symptoms first appeared in the young apical leaves. This was analogous to a typical wildtype TBSV infection but without the rapidly appearing apical necrosis (Fig. 2I). The symptoms, although milder and more chlorotic than wild-type TBSV, progressed to severe chlorosis with small necrotic spots and quickly spread to the whole plant. The accumulation of viral-specific RNA approached wild type-TBSV levels at 9 dpi (Fig. 3). In addition, virus particles with the characteristic electrophoretic mobility of TCV virions could be readily isolated from systemically infected plants (data not shown).

These results provide evidence that the role of the viral CP in enabling systemic movement of TBSV RNA likely involves virus assembly. We would like to emphasize that systemic invasion close to wild-type TBSV occurred only when the mutant RNA was able to package into virions. This suggests that formation of virus particles is an important prerequisite for normal systemic movement of TBSV. However, the possibility remains that the assembly only protects viral RNAs from degradation in plants and that some other interaction of the $\mathrm{CP}$ with an unknown host factor is what actually enables the exit of unassembled TBSV RNAs from the vascular tissue.

\section{The role of $\mathrm{p} 19$ in TBSV systemic invasion as a suppressor of PTGS.}

The intriguing observation by others (Voinnet et al. 1999) that $\mathrm{p} 19$ functions as a suppressor of PTGS prompted us to reevaluate the role of p19 in TBSV systemic infection of $N$. benthamiana in the presence and in the absence of a functional p22 movement gene. To accomplish this, we produced a deletion mutant (TB- $\Delta \mathrm{NB}$ ) in which most of the p19 and p22 coding regions were deleted. A second mutant, TBQ2, was produced in which the ORF encoding p19 was inactivated by abolishing the start codon and changing the third codon to UGA, a stop codon. These changes prevented translation of p19 while preserving translation of p22. Each mutant was inoculated onto three NT and three MP+ plants. As expected, the double deletion mutant (TB- $\Delta \mathrm{NB}$ ) failed to infect NT plants. Also, as previously observed by Scholthof and associates (1995a), systemic infection of the p19 defective mutant 
(TBQ2) initially proceeded like a wild-type TBSV infection in NT plants. TB- $\triangle \mathrm{NB}$ and TBQ2 behaved similarly on MP+ plants, showing wild type TBSV symptoms as early as 6 dpi (Fig. 2J). However, symptom development on TB- $\Delta$ NB-inoculated MP+ plants, as well as on TBQ2-inoculated NT and MP+ plants, slowed down relative to plants infected with wild-type TBSV, starting at 7 to 8 dpi. Typically, wild-type TBSV infections proceeded into apical necrosis and plant death by $14 \mathrm{dpi}$. In contrast, the symptoms of p19-defective mutants became visibly milder and the leaves became less curled as the infections approached 14 dpi (Fig. 2K). Although small necrotic areas were still seen on many leaves (Fig. 2K), the newly emerged leaves were generally much less symptomatic. The most striking result was that viral RNA accumulation in the symptomatic leaves of the TB- $\Delta \mathrm{NB}$-inoculated MP+ plants and TBQ2-inoculated plants was markedly reduced at 9 dpi (Fig. 3, lanes 6 and 7). This suggested a correlation between the onset of symptom attenuation and a reduction in viral RNA accumulation.

To verify this possibility, we directly compared the relative accumulation of viral RNAs in nontransgenic plants inoculated with wild-type TBSV transcript (T100) and the p19 mutant (TBQ2) over the period between 5 dpi and 14 dpi (Fig. 5). Eight plants were inoculated with each construct and viral RNA levels in the systemic leaves were examined daily, using RNA blot analysis. Figure 5A shows viral RNA extracted from systemic leaves at 5, 6, 7, 8, 10, and $14 \mathrm{dpi}$, with each panel containing independent samples from two T100-infected plants and two TBQ2-infected plants. The results clearly demonstrated that, while the viral RNA levels in TBQ2-inoculated plants initially approached that of T100 (5 and $6 \mathrm{dpi}$ ), they decreased progressively beginning at $7 \mathrm{dpi}$. By $14 \mathrm{dpi}$, as calculated from a dilution experiment, viral RNA levels in TBQ2-infected plants were at least 200-fold less than in wild-type TBSV-infected plants (data not shown). We conclude that the p19 defective mutant replicated as efficiently as wild-type TBSV early in infection but began to be rapidly eliminated from infected leaves within a week of infection.

The above results led us to believe that the host defense mechanism targeting viral RNAs, referred to as virus-induced gene silencing (VIGS) (Ruiz et al. 1998; Ratcliff et al. 1999), was responsible for the swift elimination of TBQ2 RNAs. VIGS is considered a form of PTGS. As observed by Hamilton and Baulcombe (1999), the presence of a 25-nt RNA species is a hallmark of PTGS. To determine if the 25-nt RNA species was also present in the TBQ2-infected plants, we processed RNA samples from both T100- and TBQ2-infected plants at 10 dpi, following the procedure of Dalmay and associates (2000a), and performed RNA blot analysis with a TBSV-specific probe. As shown in Figure 5B, the 25-nt RNA species was readily detectable in TBQ2 samples. More interestingly, the same small RNA species was present in T100 samples in an even greater amount. This suggests that the RNA-degradation step, referred to as a maintenance step by others (Ruiz et al. 1998; Voinnet et al. 2000) was not affected by p19. Instead, p19 may interfere with the production or movement of the mobile silencing signal, as proposed by Voinnet and associates (1999). Our observations extend the conclusion of Voinnet and associates (1999) that p19 not only suppresses PTGS of transgenes but also is needed for actual TBSV infection by functioning to interfere with VIGS that would otherwise clear infected leaves of replicating viral RNA and prevent sustained infection.

\section{Systemic spread of TBSV does not occur when both CP and p19 are eliminated.}

It is evident from the results presented in the previous sections that both CP and p19 have significant but distinctly dif- ferent effects on the efficiency of systemic invasion by TBSV. To further elucidate the roles of $\mathrm{CP}$ and P19 in the systemic TBSV infection in $N$. benthamiana, we made two additional mutants in which both the CP and the p19 functions were eliminated. The first was a double deletion mutant $(\mathrm{TB}-\Delta \mathrm{MA}+\Delta \mathrm{NB})$ in which the deletions in the TB- $\Delta \mathrm{MA}$ and TB- $\Delta \mathrm{NB}$ were combined. The second mutant, TBQ2-CPstop, was produced by combining the p19 translational defect in TBQ2 with the CP translational defect in TB-CPstop. None of the NT and MP+ plants inoculated with $\mathrm{TB}-\Delta \mathrm{MA}+\Delta \mathrm{NB}$ and TBQ2-CPstop showed any signs of systemic symptoms during the duration of the experiment ( $35 \mathrm{dpi}$ ). Except for the NT plants infected with $\mathrm{TB}-\Delta \mathrm{MA}+\Delta \mathrm{NB}$, the inoculated leaves of other infected plants did produce numerous chlorotic spots indicative of replication and cell-to-cell movement. Figure $2 \mathrm{~L}$ shows a leaf-inoculated with TBQ2-CPstop. The chlorotic spots on TB- $\Delta \mathrm{MA}+\Delta \mathrm{NB}$-inoculated leaves of $\mathrm{MP}+$ plants were smaller, less dense, but still visible. TBSV-specific RNAs were detectable in both TB$\Delta \mathrm{MA}+\Delta \mathrm{NB}$-inoculated leaves of $\mathrm{MP}+$ plants and TBQ2CPstop-inoculated leaves as early as $4 \mathrm{dpi}$, further demonstrating that these mutants were able to replicate and move cell to cell. We previously observed that our RNA blot analysis was not sensitive enough to detect RNA replication in single cells. Therefore, we used nontransgenic $N$. benthamiana leaves inoculated with TBQ1 as controls to illustrate the cell-to-cell movement of the other two mutants (Fig. 6, lane 3).

\section{DISCUSSION}

The results reported here demonstrate that the two viral genes CP and p19, in addition to the movement protein gene

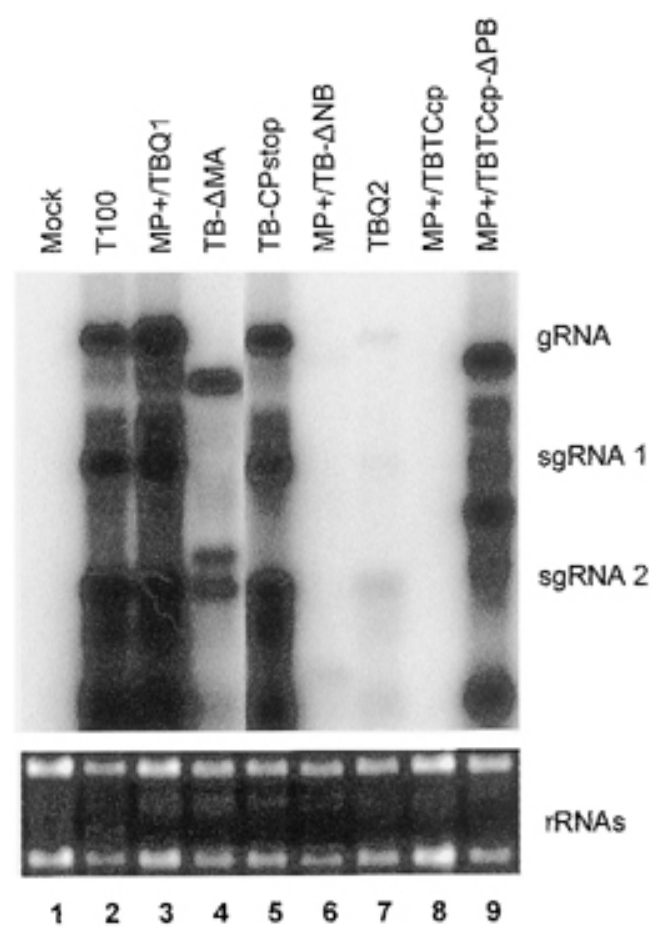

Fig. 3. RNA blot analysis of the RNAs extracted from systemic leaves of plants inoculated with different mutants. Systemically infected leaves with symptoms were selected from infected plants at 9 days postinoculation. Asymptomatic apical leaves were selected in the case of $\mathrm{MP}^{+} / \mathrm{TBTC} \mathrm{Cp}$ because no symptoms were evident at the time of the experiment. The legend above the lanes identifies the mutant inoculated. $\mathrm{MP}^{+}$before the mutant name indicates that the tissue was selected from transgenic plants. The bottom panel shows the amount of rRNAs in each lane and serves as a loading control in this and each of the remaining figures. 
$\mathrm{p} 22$, function cooperatively to promote the rapid invasion that typically leads to systemic lethal necrosis in infections caused by TBSV. We became interested in reexamining the function of $\mathrm{CP}$ in systemic movement in $N$. benthamiana because of the generally held perception that functional viral $\mathrm{CP}$ was not needed for efficient systemic spread by tombusviruses. This observation was reported for TBSV by Scholthof et al. (1993) and also was found with two other tombusviruses (Dalmay et al. 1993; McLean et al. 1993). The lack of a requirement for $\mathrm{CP}$ to invade systemically is quite an unusual property in view of the fact that all other icosahedral RNA viruses examined, including closely related members of same virus family, have an absolute requirement of the $\mathrm{CP}$ for vascular movement (Seron and Haenni 1996). An additional interesting feature of TBSV systemic movement is the growing evidence that p19 plays a more indirect role in systemic invasion and symptom development in that it most likely intensifies the viral infection by alleviating host defense responses through suppression of PTGS (Burgyan et al. 2000; Chu et al. 2000; Scholthof et al. 1995a, 1995b; Vionnet et al. 1999).

The approach we chose for reexamining the role of p19 and $\mathrm{CP}$ genes in systemic movement involved two strategies. First, we provided the TBSV cell-to-cell movement function transgenically, using a functional homologue from a closely related virus, RCNMV. In addition, we took advantage of the chimeric constructs used in our previous work (Qu and Morris 1997) in which the TBSV CP gene was replaced with a functionally equivalent $\mathrm{CP}$ gene from the structurally related TCV. These strategies permitted the construction of a series of viral $\mathrm{CP}$, chimeric $\mathrm{CP}$, and p19 mutants with replication competent genomes that could then be comparatively examined for their ability to systemically invade the plant host. Using this system, we showed that viral CP was indeed an important component of the normal systemic invasion process, as it seems that exit from the vascular tissue occurred only inefficiently in its absence. We have also provided direct evidence that the p19 gene is essential for the virus to sustain the infection by suppressing the host defense system that would otherwise lead to elimination of the viral RNAs from the infected leaf. These findings are discussed in greater detail below.

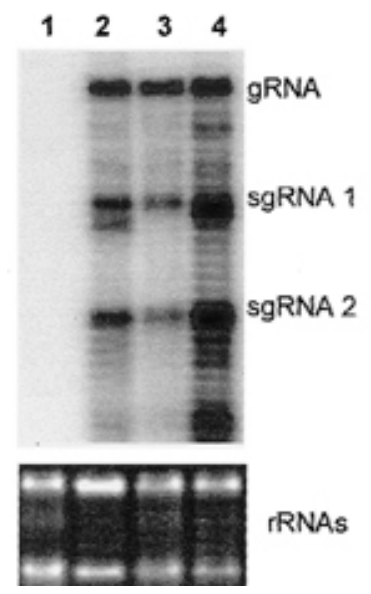

Fig. 4. RNA blot analysis of RNAs extracted from samples taken from different parts of a necrotic branch on a nontransgenic plant inoculated with the TB-CPstop mutant. The branch was dissected at 21 days postinoculation. Lane 1 , A completely asymptomatic leaf on a branch that showed obvious veinal necrosis. Lane 2, The necrotic region of the selected branch. Lane 3, An asymptomatic area from an infected leaf. Lane 4, The symptomatic area of the same leaf analyzed in lane 3.
The role of $\mathrm{CP}$ in viral systemic movement.

The general phenotype observed for TBSV CP mutants included a delay of up to 7 days in the appearance of systemic symptoms, an absence of infection of the apical region, relatively infrequent invasion of the leaves, and apparent damage to the vascular tissue. These consistent observations support the idea that the primary defect in the $\mathrm{CP}$ mutants was at the step of exit from the vascular tissue in the systemic leaves. This was evident because the CP mutants failed to invade much beyond a fairly localized area of the systemically infected leaf, usually remaining localized in tissues surrounding a primary leaf vein (class I and II veins as defined by Roberts and associates [1997], discussed below). During later stages of infection, the systemic necrosis typical of TBSV infection was generally limited to one side of larger stems and petioles that bore either mildly infected or relatively healthy leaves. Interestingly, our results on the movement of the CP-defective mutants are in agreement with field observations on the development of tomato decline disease caused by TBSV (Gerik et al. 1990). The damage of tomato decline disease is largely restricted to necrotic lesions in the stem and roots of field plants, leading to the stunting and cessation of growth of the plants. These field symptoms were shown to be temperature-dependent, a feature of TBSV infection established earlier by Hillman and associates (1985). Another similar example is the temperature-dependent movement of Brome mosaic virus (BMV), in which the virus was found almost exclusively in vascular elements when plants were grown at lower temperature (Ding et al. 1999). The temperature dependence of viral systemic spread displayed by TBSV and BMV in their natural hosts may also help explain why the degree of symptom development of CPdefective mutants differs so markedly in different studies.

The recent model of systemic movement of Potato virus $X$ (PVX) in N. benthamiana (Roberts et al. 1997) provides support for our contention that CP primarily mediates escape from the vascular elements. In that study, they examined the movement of PVX expressing green fluorescence protein (GFP) from inoculated into systemic leaves. In an elegant demonstration, they showed that PVX follows a source-sink pattern of movement in the vascular parenchyma that results in symplastic unloading of virus from primarily class III veins in the systemically invaded leaves. In their study, class I and class II veins were described as the larger midrib and primary veins in the leaf and class III veins were the smaller veins that form a veinal network directly bordering the mesophyll cells. Interestingly, they also noted that virus entry into the vascular system in inoculated leaves seemed to occur primarily through class I and class II veins. Our results suggest that TBSV moves fairly easily into the vascular system as free RNA or RNA bound with viral proteins other than $\mathrm{CP}$ in the inoculated source leaves. However, in the absence of $\mathrm{CP}$, it is unable to exit efficiently from the class III veins in the sink leaves. As a result, the viral RNA remains relatively restricted to the vascular tissue with some limited escape from class I and class II veins into spatially adjacent regions that are invaded in a cell-to-cell fashion. This model may account for the limited distribution of $\mathrm{CP}$ mutants from the vascular system of the sink leaves and the absence of invasion of the virus in primary sinks such as the shoot apex where wild-type TBSV spreads very rapidly.

Our results using the chimeric mutants expressing TCV CP permit us to speculate that the mechanism by which $\mathrm{CP}$ promotes systemic invasion and exit from the vascular elements likely involves the formation of virus particles. We base this conclusion on the observation that deletion of the genome of the chimera to a packageable size (compare mutants TBTCcp$\triangle \mathrm{PB}$ and TBTCcp) restored a near wild-type rate of systemic apical invasion to the chimeric genome that was otherwise re- 
stricted to inoculated leaves. It is generally accepted that $\mathrm{CP}$ is a fundamentally important element in the vascular movement of most viruses, but mechanisms appear to vary for different viruses. There is good evidence in support of the notion that viral assembly may be the essential requirement for translocation of many small RNA spherical and rod-shaped viruses including members of the tobamovirus, potyvirus, furovirus, sobemovirus, cucumovirus, and bromovirus genera (Seron \& Haenni 1996). There is also good evidence for assembly being essential for vascular movement of other members of the Tombusviridae family, including dianthoviruses (Vaewhongs and Lommel 1995) and carmoviruses (Cohen et al. 2000; Heaton et al. 1991).

A less likely explanation of the role of $\mathrm{CP}$ in vascular unloading is that the $\mathrm{CP}$, or a portion of the subunit, may interact specifically with a host factor to promote egress of viral RNAs from the sieve elements. Despite the suggestion that a different type of plasmodesmata may mediate trafficking into and out of sieve elements (Nelson and van Bel 1998) and the recent demonstration that a noncapsid protein of Groundnut rosette umbravirus could substitute for TMV CP to facilitate TMV systemic movement (Ryabov et al. 1999), we currently do not have any evidence to support the idea that TBSV CP interacts specifically with a host factor.

\section{The role of p19}

in systemic invasion and symptom recovery.

An unusual, movement-related function for the TBSV p19 gene was first suggested by Scholthof et al. (1995a) with the demonstration that the gene appeared to be required for systemic movement in some hosts but not in $N$. benthamiana. When the p19 gene was expressed in $N$. benthamiana using a PVX-based vector, p19 appeared to function primarily as a symptom determinant causing the characteristic necrosis typical of wild-type TBSV infections (Scholthof et al. 1995b). Interestingly, these results are very similar to those reported for gene $2 \mathrm{~b}$ mutants of Cucumber mosaic virus (CMV) by Ding and associates (1995). The demonstration that both the CMV $2 \mathrm{~b}$ protein and the TBSV p19 protein suppressed PTGS of a GFP transgene (Brigneti et al. 1998; Voinnet et al. 1999) provided a plausible mechanism by which symptom modulation could be explained. Here, we have shown that the p19 mutants do indeed allow for PTGS, which, in turn, eliminated the viral RNA from the infected plants. Therefore, the function of p19 as the suppressor of PTGS is essential for TBSV to escape this RNA-mediated host defense and invade the plant with a sustained systemic infection. The detection of the 25-nt RNA species from both wild-type TBSV and p19 mutant infected plants further define the role of p19 as likely interfering with the production or delivery, or both, of the mobile silencing signal.

We observed that the accumulation of p19 mutant viral RNA in systemically infected leaves initially reached that of wildtype TBSV but began to decline progressively as early as 7 dpi. The symptoms of infection moderated accordingly. These results also showed that the p19 mutants were not in the least compromised for replication or movement. The decline of the viral RNA level in these plants appeared to be attributable to the result of PTGS. The symptom modulation observed in the

A:
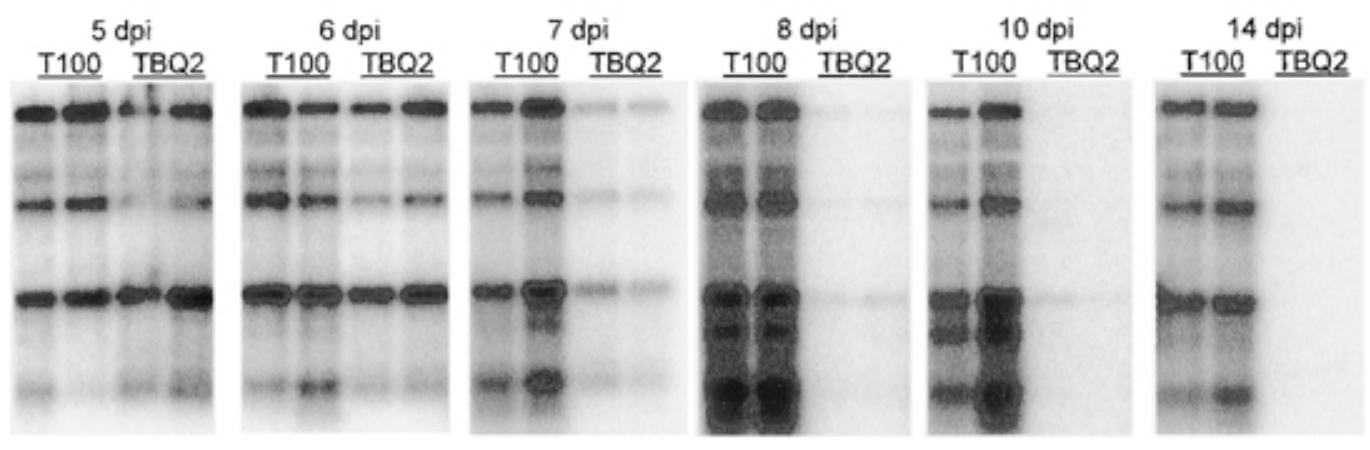

gRNA

sgRNA 1

sgRNA 2
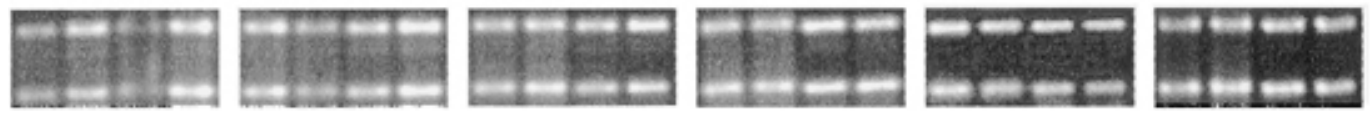

rRNAs

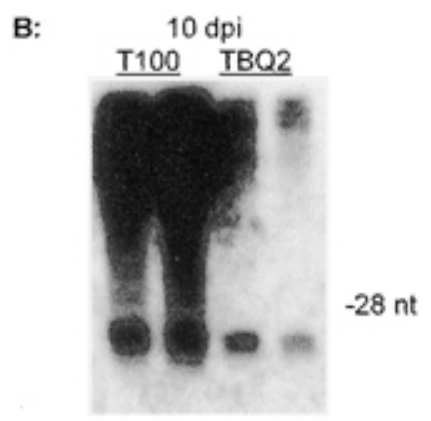

Fig. 5. A time course analysis of RNAs extracted from Nicotiana benthamiana plants inoculated with wild-type Tomato bushy stunt virus (TBSV) T100 and the p19-defective mutant TBQ2 and detection of the 25-nt RNA species. A, Eight plants were inoculated with each construct. Viral RNA levels in the systemically infected leaves were examined with RNA blot analysis. Starting at 5 days postinoculation (dpi), two T100-infected and two TBQ2-infected plants were analyzed every day. The results from samples taken at 5, 6, 7, 8, 10, and 14 dpi are presented. B, Detection of the 25-nt RNA species from the 10-dpi samples. A 28-nt TBSV-specific oligodeoxyribonucleotide was used as the size marker. 
TBQ2-infected plants is analogous to that observed in plants infected with Cauliflower mosaic virus (Covey et al. 1997) and Tomato black ring nepovirus (Ratcliff et al. 1997), a process referred to as "recovery." Interestingly, the TBQ2-infected plants showing signs of recovery were also resistant to challenge inoculation with wild-type TBSV (data not shown).

It is also worth noting here that leaves on recovered plants were never completely symptom free (Fig. 2K). Although symptom severity appeared to gradually reduce in leaves with declining viral RNA content, leaf rugosity and necrotic spots indicative of former virus replication were still evident after several weeks. It seems, therefore, that the severe necrotic symptoms associated with TBSV infection are not likely the direct result of p19 expression but rather are an indirect effect of the reduced TBSV accumulation caused by PTGS. This interpretation is consistent with several observations, including the effect of temperature on the development of TBSV symptoms in general (Hillman et al. 1985), the symptom attenuation associated with competition between genomic and defective interfering RNAs (Hillman et al. 1987), and that the viral replicase complex (p33) can directly alter the severity of necrosis (Burgyan et al. 2000).

It is becoming generally accepted that a PTGS-based host defense system targeting excess RNAs may well be induced in plants infected with most viruses (Carrington et al. 2001; Voinnet et al. 1999). Consequently, plant viruses in general are thought to encode functions that combat PTGS. The virus-encoded suppressors of PTGS have now been identified for many different viruses (Anandalakshmi et al. 1998; Brigneti et al. 1998; Kasschau and Carrington 1998; Voinnet et al. 1999, 2000). Furthermore, several virus-encoded suppressors have been determined to target various steps of the PTGS signaling pathway (Mallory et al. 2001; Voinnet et al. 2000). One plantencoded suppressor of PTGS has also been identified by its ability to interact with a viral suppressor, the HC-Pro of a potyvirus (Anandalakshmi et al. 2000). A number of other host genes have also been found to participate in this process

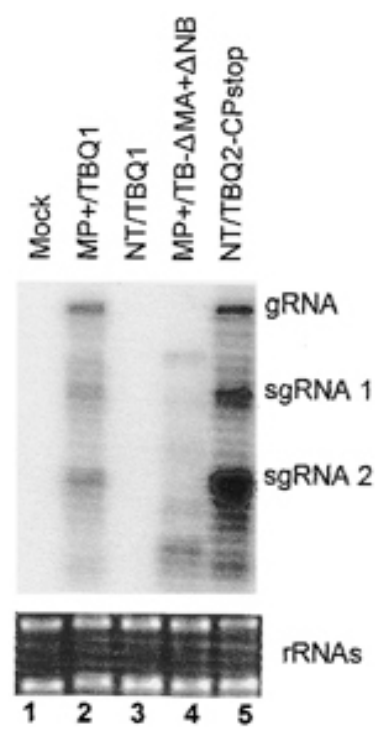

Fig. 6. RNA blot analysis of RNAs extracted from inoculated leaves of nontransgenic (NT) plants and transgenic plants expressing Red clover necrotic mosaic virus movement protein $\left(\mathrm{MP}^{+}\right)$inoculated with various single and double mutants at 4 days postinoculation. Lane 1 , Mock inoculated leaf. Lane 2, p22 defective TBQ1 inoculated $\mathrm{MP}^{+}$leaf. Lane 3, TBQ1 on an NT leaf. Lane 4 , TB- $\triangle \mathrm{MA}+\Delta \mathrm{NB}$, the double deletion mutant with defective coat protein $(\mathrm{CP}), \mathrm{p} 19$, and p22 genes on an $\mathrm{MP}^{+}$ plant. Lane 5, TBQ2-CPstop, the mutant defective in translation of both $\mathrm{CP}$ and 19 on an NT plant.
(Dalmay et al. 2000b; Fagard et al. 2000; Mourrain et al. 2000; Wu-Scharf et al. 2000).

An interesting outcome from this study has been the preliminary identification of the likely PTGS suppressor in the TCV genome. We were initially puzzled by the observation that the chimeric TBTCcp- $\triangle \mathrm{PB}$ mutant RNA was able to accumulate to very substantial levels in MP+ plants in spite of the fact that p19 was completely deleted (Fig. 3, lane 9). This result suggested that the TCV CP not only complemented the function of TBSV CP but also served as a replacement for p19, namely the suppressor of PTGS. We now have preliminary data demonstrating that TCV CP does indeed function as a suppressor of PTGS (Qu and Morris, unpublished data). This is very intriguing because the same viral CP modulates the resistance response of Arabidopsis to TCV infection (Ren et al. 2000).

\section{MATERIALS AND METHODS}

\section{Recombinant virus constructs.}

Routine molecular biological protocols were followed in making the constructs used in the current study (Sambrook et al. 1989). The point mutations in mutants TBQ1, TB-CPstop, and TBQ2 were introduced with a polymerase chain reaction (PCR) procedure, using the following oligodeoxyribonucleotides: TBQ1, 5'CAAGACCAGTTCAAGGATACTGAATAC3'; TB-CPstop, 5'ACTGGCATTGGGTTAATAAGCTGGGGCCAC3'; TBQ2, 5'CAAGTCAATAAACCTTGGAATGAGCTATACAAGGA3'. These three mutants were all sequenced to verify the existence of the desired changes. Mutant TB- $\Delta$ MA was produced by digesting the plasmid T100 with $M s c \mathrm{I}$ and AgeI, treating the digested fragments with Klenow (Gibco/BRL, Grand Island, NY, U.S.A.), and religating the larger fragment. The mutant TB- $\Delta \mathrm{NB}$ was produced similarly, except that digestion was carried out with $N c o I$ and $B s t \mathrm{BI}$.

\section{Plant materials and inoculations.}

Both nontransgenic (NT) and RCNMV MP-transgenic (MP+) N. benthamiana plants (Vaewhongs and Lommel 1995) were grown in the greenhouse. The MP+ plants looked a little smaller and paler than NT plants but otherwise appeared normal. The plants were inoculated when they had about eight fully developed leaves. The inocula were in vitro transcribed RNAs of the various constructs, diluted to $100 \mu \mathrm{g}$ per $\mathrm{ml}$ with the inoculation solution $\left(30 \mathrm{mM} \mathrm{K} \mathrm{HPO}_{4}, \mathrm{pH} 9.2 ; 50 \mathrm{mM}\right.$ glycine; $1 \%$ bentonite; $1 \%$ celite) per $\mathrm{ml}$. Two leaves of every plant were each inoculated with $20 \mu \mathrm{l}$ of the diluted RNA. In addition, all of the mutants were evaluated for replication competency in cucumber protoplasts, as described previously (Qu and Morris 1997).

\section{RNA blot analysis.}

RNAs were extracted from the infected plants using a conventional protocol (Hillman et al. 1985). The RNAs were then run on agarose gels and transformed to nylon membranes as described earlier ( $\mathrm{Qu}$ and Morris 1997). The membranes were probed with a ${ }^{32} \mathrm{P}$-labeled cDNA fragment derived from the $3^{\prime}$ noncoding region of TBSV genome.

\section{Detection of the 25-nt RNA species.}

The low-molecular-weight RNAs were isolated from the total RNA samples and separated on a denaturing polyacrylamide gel as described by Dalmay and associates (2000a). The $15 \%$ polyacrylamide (the acrylamide/bisacrlamide ratio was 29:1) gel slab contained $8 \mathrm{M}$ urea and $0.5 \times$ Tris-borate-EDTA. After separation, the RNAs were blotted to nylon membrane and hybridized to a ${ }^{32} \mathrm{P}$-labeled TBSV-specific probe. The probe was derived from a mixture of TBSV cDNA fragments smaller than $150 \mathrm{nt}$. The hybridization was carried out at $35^{\circ} \mathrm{C}$ 
with the hybridization buffer containing $50 \%$ formamide. Prior to the denaturing polyacrylamide gel electrophoresis, the small RNA samples were also separated by agarose gel electrophoresis to ensure roughly equal amounts of RNAs were used for all samples (Mallory et al. 2001).

\section{ACKNOWLEDGMENTS}

We thank S. Lommel and T. Sit for kindly providing us the seeds of the transgenic $N$. benthamiana plants expressing RCNMV MP. S. Lommel and A. White are greatly appreciated for critically reading the manuscript. We also thank S. Link and other greenhouse staff for taking care of the plants. We are very grateful to $\mathrm{S}$. Weldon for her generous help in editing the color figure. This study is supported in part by a Department of Energy grant (DE-FG03-98ER20315).

\section{LITERATURE CITED}

Anandalakshmi, R., Pruss, G. J., Ge, X., Marathe, R., Smith, T. H., and Vance, V. B. 1998. A viral suppressor of gene silencing in plants. Proc. Natl. Acad. Sci. U.S.A. 95:13079-13084

Anandalakshmi, R., Marathe, R., Ge, X., Herr, J. M., Jr., Mau, C., Mallory, A., Pruss, G., Bowman, L., and Vance, V. B. 2000. A calmodulin-related protein that suppresses posttranscriptional gene silencing in plants. Science 290:142-144.

Brigneti, G., Voinnet, O., Li, W. X., Ji, L. H., Ding, S. W., and Baulcombe, D. C. 1998. Viral pathogenicity determinants are suppressors of transgene silencing in Nicotiana benthamiana. EMBO (Eur. Mol. Biol. Organ.) J. 17:6739-6746.

Burgyan, J., Hornyik, C., Szittya, G., Silhavy, D., and Bisztray, G. 2000. The ORF1 of tombusviruses play a crucial role in lethal necrosis of virus-infected plants. J. Virol. 74:10873-10881.

Carrington, J. C., Kasschau, K. D., Mahajan, S. K., and Schaad, M. C. 1996. Cell-to-cell and long distance transport of virus in plants. Plant Cell 8:1669-1681.

Carrington, J. C., Kasschau, K. D., and Johansen, L. K. 2001. Activation and suppression of RNA silencing by plant viruses. Virology 281:1-5.

Cohen, Y., Gisel, A., and Zambryski, P. C. 2000. Cell-to-cell and systemic movement of recombinant green florescent protein-tagged turnip crinkle viruses. Virology 273:258-266.

Chu, M., Desvoyes, B., Turina, M., Noad, R., and Scholtof, H. B. 2000. Genetic dissection of tomato bushy stunt virus p19-protein-mediated host-dependent symptom induction and systemic invasion. Virology 266:79-87.

Cooper, B., Schmitz, I., Rao, A. L. N., Beachy, R. N., and Dodds, J. A. 1996. Cell-to-cell transport of movement-defective cucumber mosaic and tobacco mosaic viruses in transgenic plants expressing heterologous movement protein genes. Virology 216:208-213.

Covey, S. N., Al-Klaff, N. S., Langara, A., and Turner, D. S. 1997. Plants combat infection by gene silencing. Nature 385:781-782.

Dalmay, T., Rubino, L., Burgyan, J., Kollar, A., and Russo, M. 1993. Functional analysis of cymbidium ringspot virus genome. Virology 194:697-704.

Dalmay, T., Hamilton, A., Mueller, E., and Baulcombe, D. C. 2000a. Potato virus $\mathrm{X}$ amplicons in Arabidopsis mediate genetic and epigenetic gene silencing. Plant Cell 12:369-379.

Dalmay, T., Hamilton, A., Rudd, S., Angell, S., and Baulcombe, D. C. 2000b. An RNA-dependent RNA polymerase gene in Arabidopsis is required for posttranscriptional gene silencing mediated by a transgene but not by a virus. Cell 101:543-553.

Dasgupta, R., Garcia, B. H., II, and Goodman, R. M. 2001. Systemic spread of an RNA insect virus in plants expressing plant viral movement protein genes. Proc. Natl. Acad. Sci. U.S.A. 98:4910-4915.

De Jong, W., and Ahlquist, P. 1992. A hybrid plant virus made by transferring the noncapsid movement protein from a rod-shaped to an icosahedral virus is competent for systemic infection. Proc. Natl. Acad. Sci. U.S.A. 89:6808-6812

Ding, S. W., Li, W. X., and Symons, R. H. 1995. A novel naturally occurring hybrid gene encoded by a plant RNA virus facilitates long distance virus movement. EMBO (Eur. Mol. Biol. Organ.) J. 14:57625772.

Ding, X. S., Flasinki, S., and Nelson, R. S. 1999. Infection of barley by brome mosaic virus is restricted predominantly to cells in and associated with veins through a temperature-dependent mechanism. Mol. Plant-Microbe Interact. 12:615-623.

Dolja, V. V., Haldeman-Cahill, R., Montgomery, A. E., Vandenbosch, K. A., and Carrington, J. C. 1995. Capsid protein determinants involved in cell-to-cell and long distance movement of tobacco etch virus.
Virology 206:1007-1116.

Fagard, M., Boutet, S., Morel, J.-B., Bellini, C., and Vaucheret, H. 2000. AGO1, QDE-2, and RDE-1 are related proteins required for post-transcriptional gene silencing in plants, quelling in fungi, and RNA interference in animals. Proc. Natl. Acad. Sci. U.S.A. 97:11650-11654.

Gerik, J. S., Duffus, J. E., Perry, R., Stenger, D. C., and Van Maren, A. F. 1990. Etiology of tomato plant decline in the California desert. Phytopathology 80:1352-1356.

Giesman-Cookmeyer, D., Silver, S., Vaewhongs, A. A., Lommel, S. A., and Deom, C. M. 1995. Tobamovirus and dianthovirus movement proteins are functionally homologous. Virology 213:38-45.

Hamilton, A. J., and Baulcombe, D. C. 1999. A species of small antisense RNA in posttranscriptional gene silencing in plants. Science 286:950952.

Hearne, P. Q., Knorr, D. A., Hillman, B. I., and Morris, T. J. 1990. The complete genome structure and synthesis of infectious RNA from clones of tomato bushy stunt virus. Virology 177:141-151.

Heaton, L. A., Lee, T. C., and Morris, T. J. 1991. Point mutations in the turnip crinkle virus capsid protein affect the symptoms expressed by Nicotiana benthamiana. Virology 183:143-150.

Hillman, B. I., Morris, T. J., and Schlegel, D. E. 1985. Effects of low-molecular-weight RNA and temperature on tomato bushy stunt virus symptom expression. Phytopathology 75: 361-365.

Hillman, B. I., Carrington, J. C., and Morris, T. J. 1987. A defective interfering RNA that contains a mosaic of a plant virus genome. Cell 51:427-433.

Kasschau, K. D., and Carrington, J. C. 1998. A counter-defensive strategy of plant viruses: Suppression of posttranscriptional gene silencing. Cell 95:461-470.

Li, W.-Z., Qu, F., and Morris, T. J. 1998. Cell-to-cell movement of turnip crinkle virus is controlled by two small open reading frames that function in trans. Virology 244:405-416.

Mallory, A. C., Ely, L., Smith, T. H., Marathe, R., Anandalakshmi, R., Fagard, M., Vaucheret, H., Pruss, G., Bowman, L., and Vance, V. B. 2001. HC-Pro suppression of transgene silencing eliminates the small RNAs but not transgene methylation or the mobile signal. Plant Cell 13:571-583.

McLean, M. A., Hamilton, R. I., and Rochon, D. M. 1993. Symptomology and movement of a cucumber necrosis virus mutant lacking the coat protein protruding domain. Virology 193:932-939.

Mourrain, P., Beclin, C., Elmayan, T., Feuerbach, F., Godon, C., Morel, J.-B., Jouette, D., Lacombe, A.-M., Nikic, S., Picault, N., Remoue, K., Sanial, M., Vo, T.-A., and Vaucheret, H. 2000. Arabidopsis SGS2and SGS3 genes are required for posttranscriptional gene silencing and natural virus resistance. Cell 101:533-542.

Nelson, R. S., and van Bel, A. J. E. 1998. The mystery of virus trafficking into, through, and out of the vascular tissue. Prog. Bot. 59:476-533.

Oster, S. K., Wu, B., and White, K. A. 1998. Uncoupled expression of P33 and P92 permits amplification of tomato bushy stunt virus RNAs. J. Virol. 72:5845-5851.

Qu, F., and Morris, T. J. 1997. Encapsidation of turnip crinkle virus is defined by a specific packaging signal and RNA size. J. Virol. 71:14281435 .

Rao, A. L. N., Cooper, B., and Deom, C. M. 1998. Defective movement of viruses in the family Bromoviridae is differentially complemented in Nicotiana benthamiana expressing tobamovirus or dianthovirus movement proteins. Phytopathology 88: 666-672.

Ratcliff, F., Harrison, B. D., and Baulcombe, D. C. 1997. A similarity between viral defense and gene silencing in plants. Science 276:15581560 .

Ratcliff, F., MacFarlane, S. A., and Baulcombe, D. C. 1999. Gene silencing without DNA: RNA-mediated cross-protection between viruses. Plant Cell 11:1207-1215.

Reade, R., Delroux, K., MacDonald, K., Sit, T. L., Lommel, S. A., and Rochon, D. 2001. Spontaneous deletion enhances movement of a cucumber necrosis virus-based chimera expressing the red clover necrotic mosaic virus movement protein gene. Mol. Plant. Path. 2:13-25.

Ren, T., Qu, F., and Morris, T. J. 2000. HRT gene function requires interaction between a NAC protein and viral capsid protein to confer resistance to turnip crinkle virus. Plant Cell 12:1917-1925.

Roberts, A. G., Simon, S. C., Roberts, I. M., Denton, A. M. P., Turgeon, R., and Oparka, K. J. 1997. Phloem unloading in sink leaves of Nicotiana benthamiana: Comparison of a fluorescent solute with a fluorescent virus. Plant Cell 9:1381-1396.

Ruiz, M. T., Voinnet, O., and Baulcombe, D. C. 1998. Initiation and maintenance of virus-induced gene silencing. Plant Cell 10:937-946.

Ryabov, E. V., Robinson, D. J., and Taliansky, M. E. 1999. A plant virusencoded protein facilitates long-distance movement of heterologous viral RNA. Proc. Natl. Acad. Sci. U.S.A. 96:1212-1217.

Sambrook, J., Fritsch, E. F., and Maniatis, T. 1989. Molecular Cloning: A 
Laboratory Manual. 2nd ed. Cold Spring Harbor Laboratory Press. Cold Spring Harbor, NY, U.S.A.

Schaad, M. C., and Carrington, J. C. 1996. Suppression of long distance movement of tobacco etch virus in a nonsusceptible host. J. Virol. 70:2556-2561.

Scholthof, H. B., Morris, T. J., and Jackson, A. O. 1993. The capsid protein gene of tomato bushy stunt virus is dispensable for systemic movement and can be replaced for localized expression of foreign genes. Mol. Plant-Microbe Interact. 6:309-322.

Scholthof, H. B., Scholthof, K.-B., Kikkert, M., and Jackson, A. O 1995a. Tomato bushy stunt virus spread is regulated by two nested genes that function in cell-to-cell movement and host-dependent systemic invasion. Virology 213:425-438.

Scholthof, H. B., Scholthof, K.-B., and Jackson, A. O. 1995b. Identification of tomato bushy stunt virus host-specific symptom determinants by expression of individual genes from a potato virus $\mathrm{X}$ vector. Plant Cell 7:1157-1172.

Seron, K., and Haenni, A.-L. 1996. Vascular movement of plant viruses. Mol. Plant-Microbe Interact. 9:435-442.

Szittya, G., and Burgyan, J. 2001. Cymbidium ringspot tombusvirus coat protein coding sequence acts as an avirulent RNA. J. Virol. 75:24112420 .

Taliansky, M. E., and Garcia-Arenal, F. 1995. Role of cucumovirus capsid protein in long-distance movement within the infected plant. J. Virol. 69:916-922.

Vaewhongs, A. A., and Lommel, S. A. 1995. Virion formation is required for the long-distance movement of red clover necrotic mosaic virus in movement protein transgenic plants. Virology 212:607-613.

Voinnet, O., Pinto, Y. M., and Baulcombe, D. C. 1999. Suppression of gene silencing: A general strategy used by diverse DNA and RNA viruses of plants. Proc. Natl. Acad. Sci. U.S.A. 96:14147-14152.

Voinnet, O., Lederer, C., and Baulcombe, D. C. 2000. A viral movement protein prevents spread of the gene silencing signal in Nicotiana benthamiana. Cell 103:157-167.

Wu-Scharf, D., Jeong, B.-R., Zhang, C., Cerutti, H. 2000. Transgene and transposon silencing in Chlamydomonas reinhardtii by a DEAH-box RNA helicase. Science 290:1159-1162.

Zhang, G., Slowinski, V., and White, K. A. 1999. Subgenomic mRNA regulation by a distal RNA element in a (+)-strand RNA virus. RNA $5: 550-561$ 